\title{
Knowledge-Based Approach to Assembly Sequence Planning for Wind-Driven Generator
}

\author{
Meiping Wu, Yi Zhao, and Chenxin Wang \\ School of Mechanical Engineering, Jiangnan University, Wuxi, Jiangsu, China \\ Correspondence should be addressed to Meiping Wu; wmp169@hotmail.com
}

Received 9 June 2013; Accepted 26 July 2013

Academic Editor: Jun Wang

Copyright (C) 2013 Meiping Wu et al. This is an open access article distributed under the Creative Commons Attribution License, which permits unrestricted use, distribution, and reproduction in any medium, provided the original work is properly cited.

\begin{abstract}
Assembly sequence planning plays an essential role in the manufacturing industry. However, there still exist some challenges for the research of assembly planning, one of which is the weakness in effective description of assembly knowledge and information. In order to reduce the computational task, this paper presents a novel approach based on engineering assembly knowledge to the assembly sequence planning problem and provides an appropriate way to express both geometric information and nongeometric knowledge. In order to increase the sequence planning efficiency, the assembly connection graph is built according to the knowledge in engineering, design, and manufacturing fields. Product semantic information model could offer much useful information for the designer to finish the assembly (process) design and make the right decision in that process. Therefore, complex and lowefficient computation in the assembly design process could be avoided. Finally, a product assembly planning example is presented to illustrate the effectiveness of the proposed approach. Initial experience with the approach indicates the potential to reduce lead times and thereby can help in completing new product launch projects on time.
\end{abstract}

\section{Introduction}

Since the 1980s, much progress has been made to generate feasible assembly sequences for complex assemblies. However, there still exist difficulties in automatic generation of good assembly sequences. For example, assembly work has a close relation to engineer's knowledge and product's design information, but due to the technologicat status of product information modeling much information relevant to assembly design could not be described and stored in product model and, obviously, could not be transferred from one design stage to another [1]. Due to the lack of supporting information in product model, it is difficult for engineers to search for good assembly sequences in a large number of possible assembly sequences [2].

However, over the past several years, driven by demand of industrial applications, solutions of supporting interactive assembly planning systems other than the automatic generation of assembly sequences have been developed. Basically, assembly sequence planning consists of two major activities: assembly modeling and assembly sequence generation. The competence of an assembly planning depends on the way by which the assembly information is modeled. Thus, it has an important application value to develop a good assembly modeling method to assembly sequence planning. Assembly model not only is used to express structure and mutual constraint relation but also is a basic carrier of expressing product information. Therefore, assembly modeling and assembly sequence planning become a focused research hotspot in automation assembly fields.

In this paper, an assembly modeling method and an assembly sequence planning method are proposed, and their capability to assist the generation of a feasible and optimum assembly sequence is verified effectively.

\section{Related Work}

2.1. Assembly Model. Since the end of the 1970s, numerous specialist and scholars have developed a series of approaches to assembly model.

ISO 10303-Part 44 [3] provides some limited assembly design representations that capture the assembly structure and the kinematic joint information. The assembly model presented establishes a neutral representation of assemblies of 
products, which are composed of sets of components. In this model, the complete products are called assemblies, and the components at the lowest levels in the assemblies are called parts. The model focuses on the hierarchy of the product and on the position and orientation between parts. One of the primary features defined in ISO 10303 is that it provides a data modeler to generate all kinds of product data structures, parts list using the same primitive entities. However, it should be noted that ISO 10303 does not adequately take in hand the following.

(1) The relationship among different product definitions for the same product: for example, the relationship of a product definition for a component in a preliminary design to a corresponding product definition for the same component in a detailed design is not captured.

(2) The decisions made and their rationale, for the entire product life cycle: ISO working Group [4] has proposed to enhance the STEP's assembly representation. In their proposal, they have defined detailed geometric information not only for hierarchical relationship but also for peer-to-peer relationships among component parts via assembly features. Geometric constraints among component parts at the detailed geometric element level are also enabled. They have included more information on component association and included detailed information about appropriate assembly features involved in component associations, although the proposal outlined the possible applications of the proposed assembly representation in four areas: kinematic analysis of assemblies, animation of assemblies, assembly/disassembly process planning, and tolerance analysis and synthesis. However, these models are all designed based on CAD environment, and they have only static descriptions of an assembly and little has been done in terms of developing standard representations that specify assembly information and knowledge. An assembly information model contains information regarding parts and their assembly relationships. Hence, we wish to emphasize the nature and information requirements for these part features and for these assembly relationships. Furthermore, we need to concentrate on the evolution of their corresponding information models during the conceptual and detailed design stages. In this paper, we propose an integrated information model for assembly representations.

2.2. Assembly Planning. Traditionally, the design for product assembly has focused mainly on the relations among product parts. This information shows only the relative positions of the product components but does not delineate the assembly sequence of the product components. In the direct assembly approach, Bourjault [5] presented the liaisons diagram, which is a graph devised to represent an assembly. The graph uses the nodes to represent the components of assembly and the arcs to establish the relations between the components. Based on this diagram, a list of "Yes/No" questions are generated, and by question-and-answer procedure we obtain the precedence constraint among the components of the final product. However, the number of questions in Bourjault's procedure grows exponentially as the part number in the assembly increases. De Fazio and Whitney [6] simplified Bourjault's procedure by reducing question count to twice the number of liaisons in the assembly. Similarly, Homem de Mello and Sanderson [7] used a representation of the directed graph of feasible assembly sequences.

In the disassembly or decomposition approach, the feasible assembly sequence is the inverse of the disassembly sequence if there is a feasible operation in the disassembly. Huang and Lee [8] proposed a symbolic representation scheme to represent the product assembly operation from the point of view of disassembling the product by generating possible disassembly sequences.

In this work, we analyzed the demands and characteristics of assembly model based on multiple-view feature modeling, studied the method of expressing assembly relation to the assembly connection graph including the engineering assembly semantics, and presented the constraint description method of assembly relation. Assembly sequence generation method based on the knowledge was studied in detail. The method was applied to National 863 project-the key components development of wind-driven generator to reduce lead times and to ensure completing new product launch projects on time.

\section{Assembly Sequence Planning Based on the Knowledge}

\subsection{Assembly Modeling}

3.1.1. Part Information Model. The foundation of assembly modeling is appropriately expressing parts information. Current available graphics processing hardware mainly support the generation of the triangle drawing since triangle patch model can be readily treated in model display and collision detection.

Part information model is the arrangement of the part level, the feature level, and the geometry level, as shown in Figure 1. The part level involves total information of the part, such as the name, number, batch size, material name, material trademark, and performance, the name of designer, and design date. The feature level includes the shape, precision, tolerance, roughness, and material heat treatment. The geometry level represents geometry and topology information of point, line, and surface, which are basic units of the solid modeling system.

3.1.2. Assembly Information Model. Here, semantic abstraction expression of assembly information is integrated by using three levels: concept/functional, structural, and part/ feature.

As shown in Figure 2, there are several interlocked stages in product design based on assembly semantics modeling. In the early stage of schematic design and functional decomposition, an abstract based on function is initially generated by a designer in his or her mind to attempt to fulfill all 


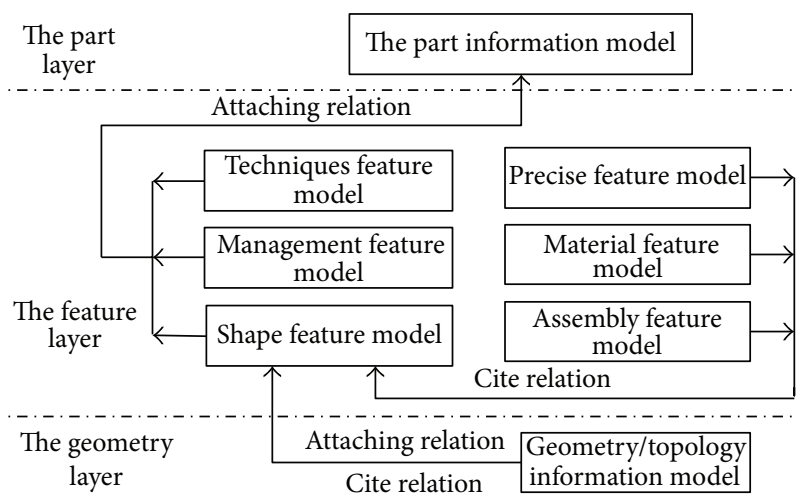

FIgURE 1: Part information model.

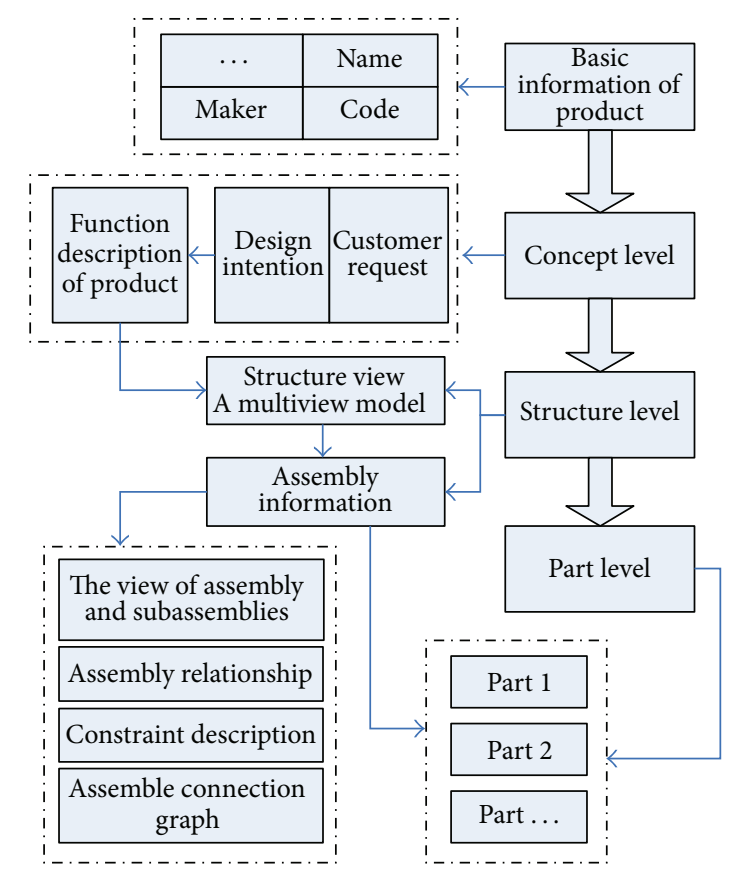

FIgURE 2: The structure of assembly model.

the requirements demanded by the targeted product. The result of this stage will yield the concept then lead to the first draft of a prototype product. Specifically, by decomposing the initial function into highly conceptual subfunctions in a step-by-step manner, prototype structure of the product will logically appear. In the following step, based on the initial structure of the product, the necessary part characteristics such as form, precision, and assembly features can be organized in hierarchical structure. Simultaneously, shortcomings can be promptly kicked back to the designer for redesign based on important factors of the product till all of the product functional requirements are rewarded.

3.2. Assembly Connection Graph. The assembly connection graph has two types of arcs with nondirected- and directedto-link-arcs nodes in order to decrease the space required for candidate assembling targets and increase optimization quality of the planned scheme. An arc represents a constraint relationship between components. Leaf nodes in the same way represent an independent subassembly or fastener, and midterm nodes represent an assembly operator. A nondirected arc represents a geometric constrain, for instance, coplane, cocentric, surface mating, and so forth, and the counterpart of a directed arc represents assembly sequence constraint, that is, an assembling sequence liaison constraint.

For instance, if the direction of the arc is toward node $j$ from node $i$, then that means component $i$ should be assembled after component $j$. As a result of the cooperation of arcs and nodes in the graph, we can continue to merge two suitable independent sub-assemblies into one higher level but more complicated subassembly until the root node is reached, that is, product model. The graph mathematic formula is given as follows:

$$
M=\{N, E, D E\},
$$

where $N$ is the node, which represents the object of an independent component, $E$ is the nondirected arc, which represents the geometric constraint relationship between two parts, and $D E$ is a directed arc, which represents the assembly sequence liaison constraint between two parts.

The procedure of creating a subassembly sequence with the assembly connection graph method employs the following four steps.

(1) Select target leaf nodes from the hierarchical model to be assembled.

(2) According to geometric constraint information of each part/component from the product assembly model, geometry constraint relationship among parts/components can be obtained from the nondirected arc.

(3) Defining assembling liaison constraint relationship between parts/components by adding directed arcs to the nondirected graph using interactive operations and generating a complete assembly connection graph of assembly sequence.

(4) Simulating the assembly sequence dynamically and evaluating the defined scheme. Simulation functions are described in application instances. Since many assembly sequences share common subsequence, attempts have been made to create more compact representation that can encompass all assembly sequences. Therefore, the works in these fields are graph-based approaches that represent all feasible assembly sequences.

From the construction process of the assembly connection graph, we can conclude that direction assembly connection graph including assembly semantic can be expressed by liaison matrix of the directed graph.

We know that a liaison always exists between a pair of parts or superparts if one part or superpart constrains the freedom of motion of the other by a direct contact or a near contact. 
3.3. Contact Matrices. The contact matrices are used to determine whether there are contacts between parts in the assembly state. These matrices are represented by a contact condition between a pair of parts. According to the assembly connection graph, the contact matrix can be represented by $L^{e}$, where $e$ is the total number of components in the assembly, for $2 \leqslant e \leqslant n$. The contact matrix $L^{e}$ of assembly planning is defined as follows.

Part number:

$$
1,2,3, \ldots n
$$

$$
L^{e}=\left[\begin{array}{cccc}
0 & j & j & j \\
i & 0 & j & j \\
i & i & 0 & j \\
i & i & i & 0
\end{array}\right] \quad \text { part number: } 1,2, \ldots, n
$$

where the characteristics of the contact matrix are described as follows:

(1) $l_{i, j}=1$, if there is contact relation between the part and part $_{j}$ in the assembly planning. Otherwise, $l_{i, j}=$ 0 .

(2) The rows of matrix represent the contact relations between the components. When $i=j, L^{e}$ represents the main diagonal consisting of its entries, then $l_{i, j}=$ 0 .

(3) The matrix $L^{e}$ is a square and symmetric matrix, so each $l_{i, j}=l_{j, i}$.

Using the ACG method, we can establish the total entries quantity $(L)$ of requirement database, which is defined as follows:

$$
L=\frac{n^{2}-n}{2} \quad n \geq 2
$$

where $n$ is the total quantity of primitive components in an assembly planning process. The ACG method of assembly sequence planning only requires the total quantity of database shown in the upper (or lower) triangular region entries of (1), which are comprised of relatively small data quantities and data contents consisting of 0 or 1 logic signs.

3.4. Generation of Feasible Subassemblies. A complicated product is constituted of tens or even hundreds of components. The completed connection graph of components is very complicated. Using a comprehensive graph to express the entire parts organization scheme will cause management difficulty and increases cost. For this reason, selecting feasible subassemblies of the complicated product must be based on evaluation factors such as stability of subassemblies, reliability of operation, and contact complexity between parts, in order to establish a simplified and standardized method of evaluation. This section presents an expression method for the parts and subassemblies merging to build a connection graph that is easily expressible for a complicated product.

3.4.1. Assembly Knowledge Representation. How to represent assembly knowledge and store it in the computer is the basic problem in the assembly sequence planning. The excellent methods can simplify computer processing. We generalized assembly features and built the knowledge representations. the knowledge representations of the part are as follows:

$\langle$ part parameters $\rangle::=(\langle$ basic parameters $\rangle,\langle$ geometry parameters $\rangle,\langle$ special parameters $\rangle)$

$\langle$ basic parameters $\rangle::=(\langle$ part name $\rangle,\langle$ part type $\rangle$, $\langle$ drawing number $\rangle$, 〈weight $\rangle,\langle$ material $\rangle,\langle$ color $\rangle)$

$\langle$ geometry parameters $\rangle::=$ (〈total character $\rangle,\langle$ key surface representation $\rangle$, 〈end surface set $\rangle$ )

$\langle$ special parameters $\rangle::=(\langle$ joint part special parameters $\rangle \mid$ (common part special parameters $\rangle)$

$\langle$ joint part special parameters $\rangle::=(\langle$ joint type $\rangle,\langle$ joint direction $\rangle$, 〈maximum joint length $\rangle)$

$\langle$ part name $\rangle::=$ character string

$\langle$ part type $\rangle::=(\langle$ joint part type $\rangle \mid\langle$ common part type $)$

$\langle$ drawing number $\rangle::=$ character string

$\langle$ weight $\rangle::=$ positive real number

$\langle$ material $\rangle::=$ (steel) $\mid$ (aluminum) $\mid$ (iron) $\mid$ (others)

$\langle$ color $\rangle::=$ (blue) $\mid$ (green) | (red) | (others)

$\langle$ total characteristic $\rangle::=(\langle$ external $\rangle,\langle$ interior $\rangle)$

$\langle$ key surface representation $\rangle::=(\langle$ key surface $\rangle,\langle$ the characteristic set $\rangle$ )

$\langle$ joint part type $::=$ bolt/|screw//rivet/|pin/|nut/ |bearing/gear/others/

$\langle$ joint method $\rangle::=0$ (joint with direction) $\mid 1$ (joint without direction)

$\langle$ joint direction〉::= vector

$\langle$ maximum joint length $\rangle::=$ positive real number.

3.4.2. Generation Assembly Planning Base on Precedence Constraints. On the assemble sequence planning field, many algorithms have been proposed for finding the entire set of possible assembly sequences [6-8]. However, many of these possible assembly sequences are unsuitable and some completely unusable. Selecting the best assembly sequence (or several feasible sequences) out of numerous possible assembly sequences is of critical importance. Nevertheless, when using previous proposed methods to account for product characteristic shifts or different production environments, it is difficult to directly yield the feasible assembly sequences. This section proposes the key evaluation factors of the product's characteristics and production operation environment to construct the precedence relationships and identify the feasible assembly sequences.

3.4.3. Assembly Precedence Relations. The assembly precedence relationships specify a partial ordering of the product assembly process. In general, the engineer cannot easily list all the relations on the assembly sequence of the product at the start of the planning stage. However, precedence relationships 


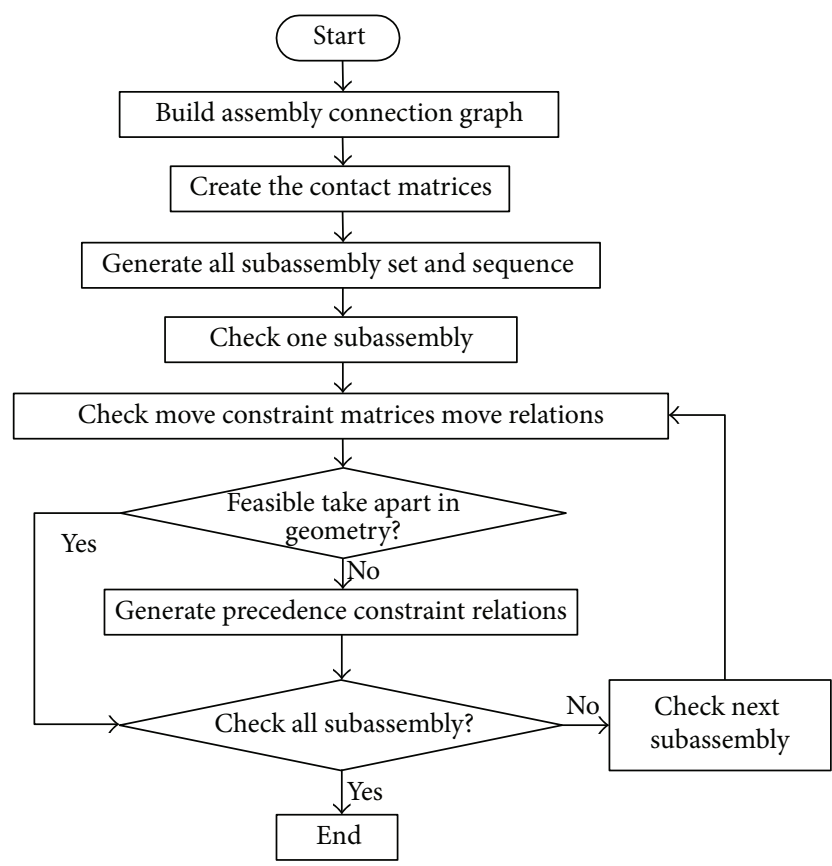

FIGURE 3: The flow chart of generating geometry precedence constraint.

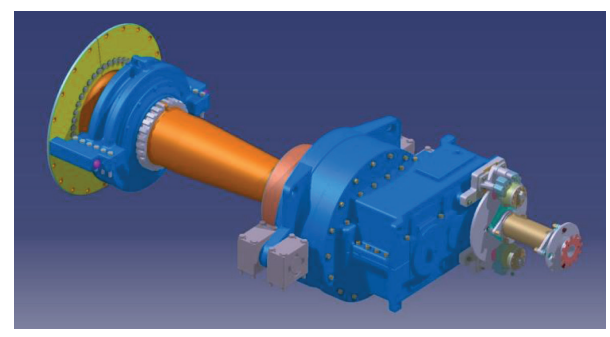

FIGURE 4: Assembly model of the transmission system.

can be powerful tools to help find the feasible sequences in assembly planning of complex product. The focal point is how to retrieve and utilize precedence constraint relationships to find feasible sequences of assembly parts in this section.

Precedence constraint relationships on local specified subassemblies can act as a guide when seeking feasible or even optimal sequences conforming to the constraint relations.

(1) Geometry Precedence Constraint. Geometry precedence constraint that cannot be violated in the assembly process is determined by geometry shape and mutual position between parts.

Move characteristic represents the possibility which the part can be disassembled in the direction. We can decide the feasible disassembly direction, according to the motion characteristic. If the part cannot be disassembled in some direction, we can construct the geometry precedence constraint. As shown Figure 3 is the flow chart of geometry precedence constraints.

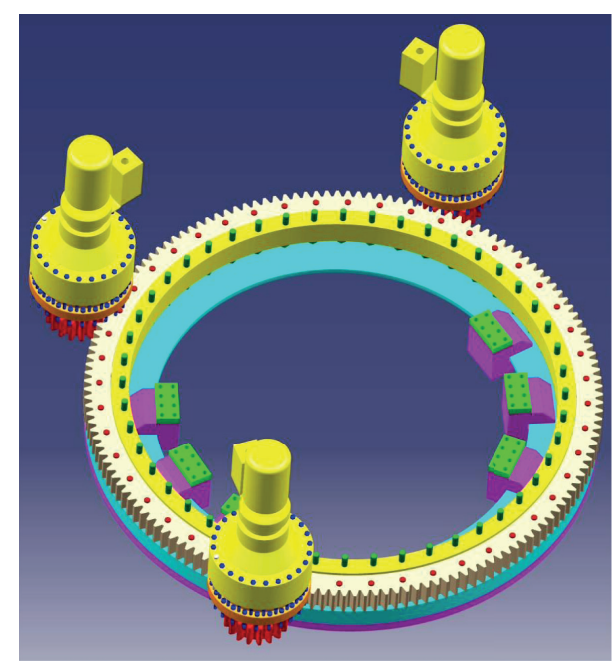

Figure 5: Assembly model of the deviation system.

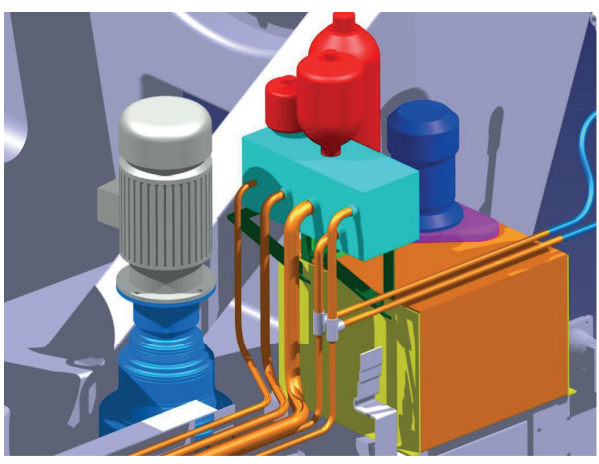

Figure 6: Assembly model of the hydraulic system.

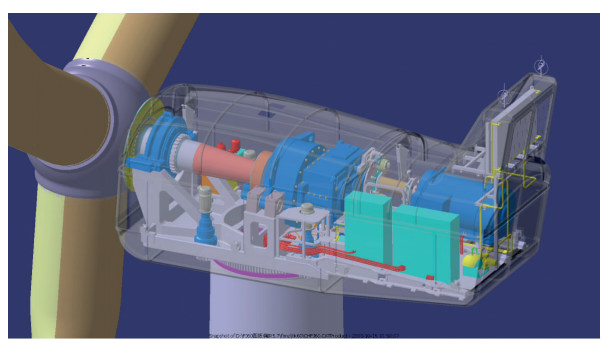

FIGURE 7: Assembly model of wind-driven generator.

(2) Special Part Precedence Constraint. Some standard part has special mandate in the assembly process, such as o-rings, spring, and bearing. For example, the spring was regarded as a rigid element in analysis, but in assembly, it was defined as two types of statue: one is the natural statue and another is under compression. Designers consider final assembly state and decide the precedence constraints relations.

Boring is the part in common mechanical product. The boring of different location, structure type, and mate mandate the use of different assembly methods.

(3) Process Precedence Constraint. Process precedence constraint was constructed by exact structure, concurrent assembly situation, and experience. These precedence constraint 


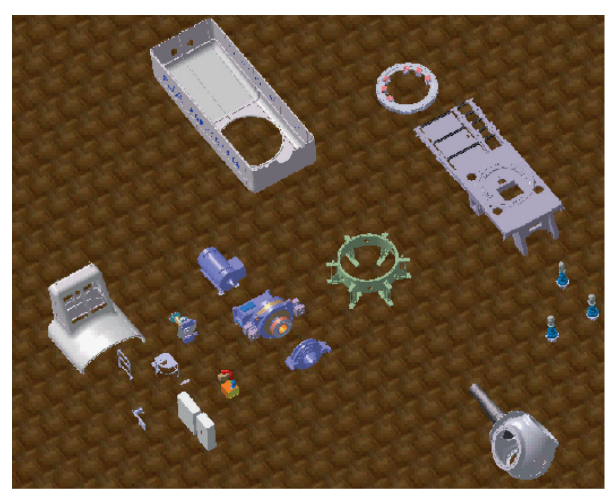

(a)

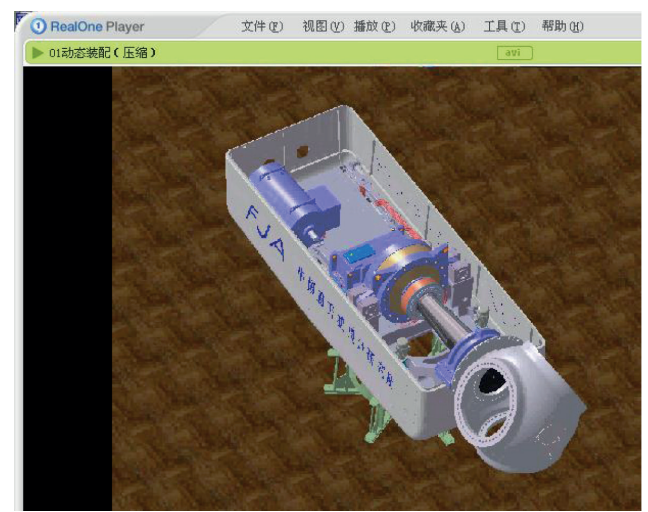

(c)

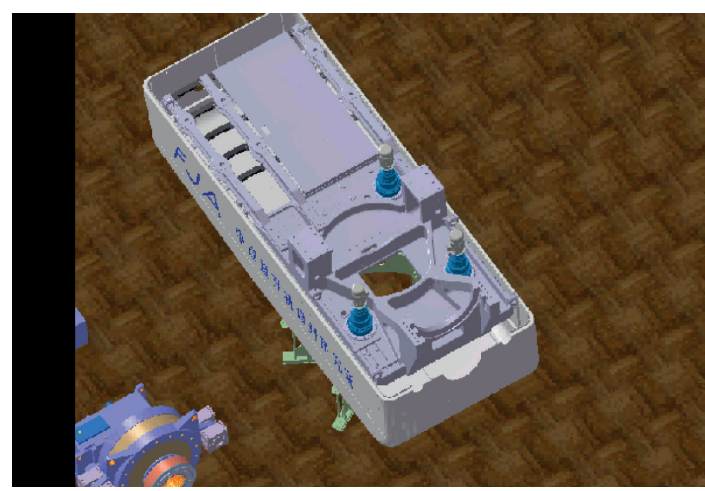

(b)

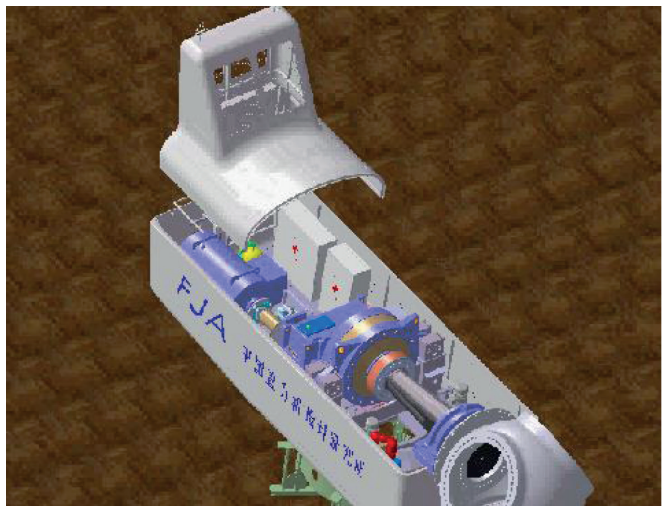

(d)

FIGURE 8: Assembly simulation of the wind-driven generator.

relations cannot be detected by geometry precedence constraint automated, so we build the process precedence constraint according to experience and process demands.

\section{The Application Instances}

4.1. Assembly Model of Transmission System. The main components of the transmission system are shown as follows: main shaft bearing, principal axis, speed-increasing gear, mechanical antiskid braking system, and high-speed shaft. Figure 4 shows the assembly model of the transmission system.

4.2. Assembly Model of Deviation System. The main components of the deviation system are shown as follows: deviation electric motors and reducer, pivoting support, and brake. Figure 5 is the assembly model of the deviation system. The parameters of deviation system are as follows: deviation rate: $0.4^{\circ}$ s. the precise: $\pm 5^{\circ}$; the sensitivity: $\pm 20^{\circ}$.

4.3. Assembly Model of Hydraulic System. Figure 6 shows the assembly model of the hydraulic system. The parameters of hydraulic system are given away as follows: the power of motor of hydraulic pump: $2.2 \mathrm{~kW}$; the system flow rate: $5.2 \mathrm{~L} / \mathrm{min}$; the sensitivity: $\pm 20^{\circ}$; the system work pressure: 10.5 16.0 Mpa.
4.4. Assembly Model of the Wind-Driven Generator. At the program design stage, we have revised and coordinated the component and system overall again and again. According to mentioned structure, function, conformity, manufacturing process, and overall coordination, check and examine the rationality of the overall system, and determine overall design program, and construct the assembly model of the winddriven generator. Figure 7 shows the overall assembly model of the wind-driven generator.

4.5. Assembly Planning Simulation of the Wind-Driven Generator. Using the previously mentioned method, we construct the assembly sequence of the wind-driven generator. Furthermore, use media techniques to simulate the assembly process and display the whole assembly process intuitively. The assembly process simulation was shown in Figure 8.

\section{Conclusion}

The method proposed by the assembly connection graph of including assembly semantics is studied in this paper. The assembly connection graph (ACG) including engineering assembly semantics proposed provides an appropriate way to express both geometric information and nongeometric knowledge. Product semantic information model could 
offer much useful information for the designer to finish the assembly design and make the right decision. Furthermore, knowledge-base approach to assembly sequence planning was studied in detailed. The application of the approach fast speed of research and development, and insure the complete of the National 863 project on time.

\section{Nomenclature}

ACG: Assembly connection graph

VA: Virtual assembly

AM: Assembly modeling

ASP: Assembly sequence planning.

\section{Acknowledgments}

This work is supported by Jiangsu Province Talented Person Project under Grant no. 07-D-023, supported by the SelfDetermined Research Program of Jiangnan University under Grant no. JUSRP20907, and National Natural Science Foundation of China (Grant no. 51275210).

\section{References}

[1] Z. Xiaoming and D. Pingan, "A model-based approach to assembly sequence planning," International Journal of Advanced Manufacturing Technology, vol. 39, no. 9-10, pp. 983-994, 2008.

[2] Q. Su, "A hierarchical approach on assembly sequence planning and optimal sequences analyzing," Robotics and ComputerIntegrated Manufacturing, vol. 25, no. 1, pp. 224-234, 2009.

[3] ISO, STEP ISO, 10303-Industrial Automation Systems and Integration Product Data Representation and Exchange-Part 44: Integrated Resources: Product Structure Configuration, ISO, Geneva, Switzerland, 1994.

[4] N. Sugimura, ISO/CD, 10303-109, Product Data Representation and Exchange: Integrated Application Resource: Kinematic and Geometric Constraints For Assembly Models, ISO, Geneva, Switzerland, 2002.

[5] A. Bourjault, Contribution a une approche methodologique de l'Assemblage Automatise: Elaboration Automatique des Sequences Operatories [Ph.D. thesis], Universite de Franche-Comte, Besançon, France, 1984.

[6] T. L. De Fazio and D. E. Whitney, "Simplified generation of all mechanical assembly sequences," IEEE Journal of Robotics and Automation, vol. 3, no. 6, pp. 640-658, 1987.

[7] L. S. Homem de Mello and A. C. Sanderson, "Representations of mechanical assembly sequences," IEEE Transactions on Robotics and Automation, vol. 7, no. 2, pp. 211-227, 1991.

[8] Y. F. Huang and C. S. G. Lee, "Precedence knowledge in feature mating operation assembly planning," in Proceedings of the IEEE International Conference on Robotics and Automation, pp. 216221, May 1989. 


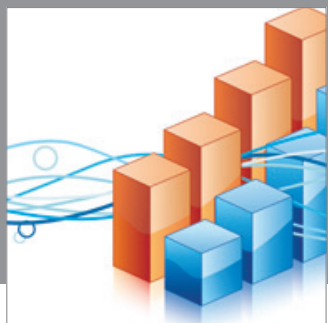

Advances in

Operations Research

mansans

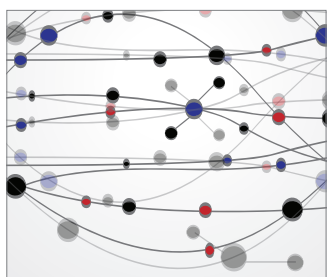

The Scientific World Journal
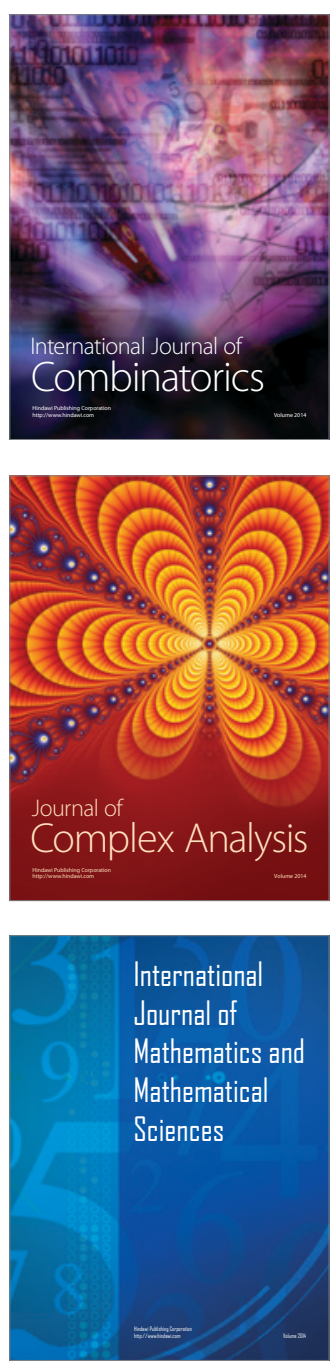
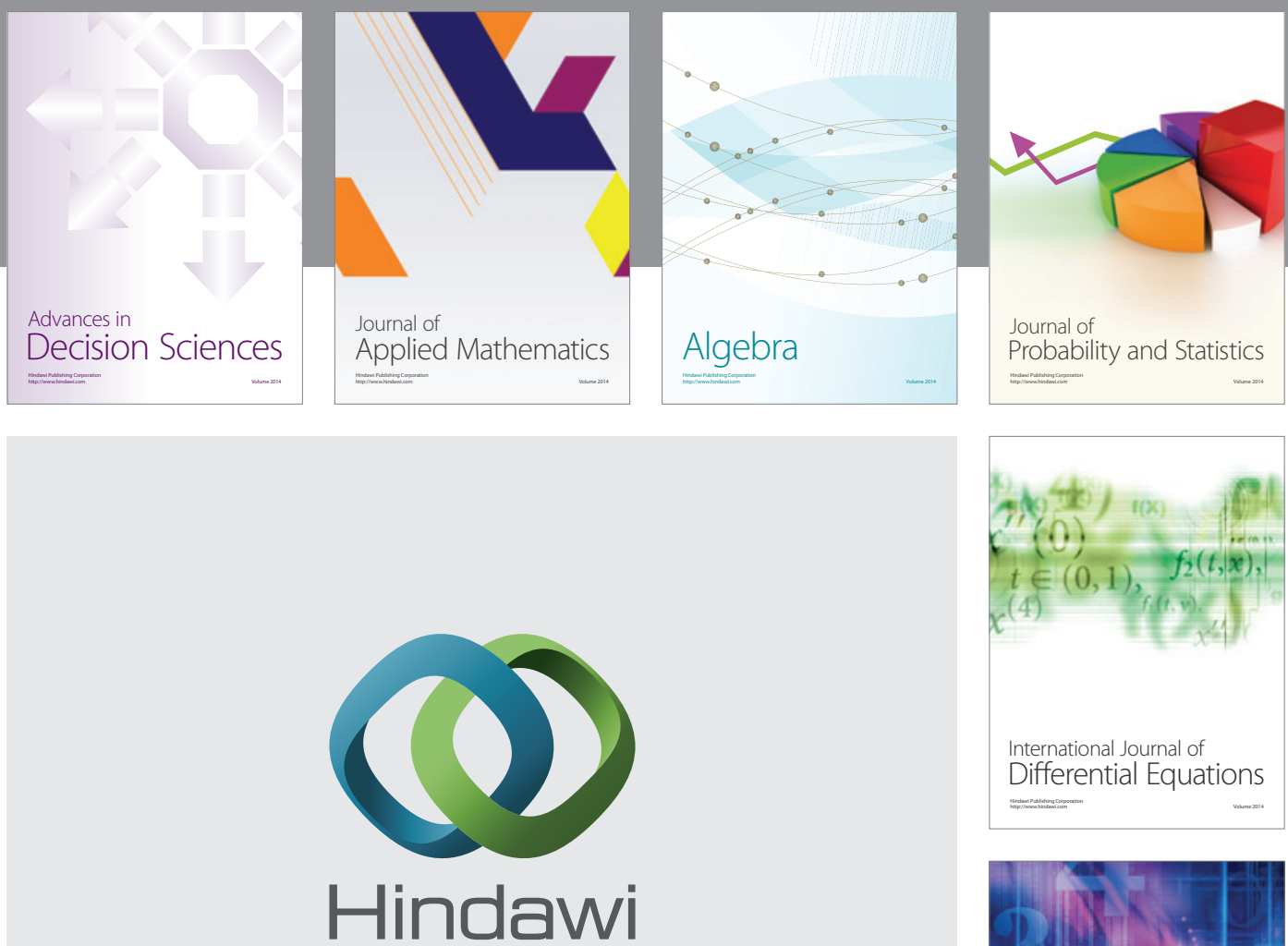

Submit your manuscripts at http://www.hindawi.com
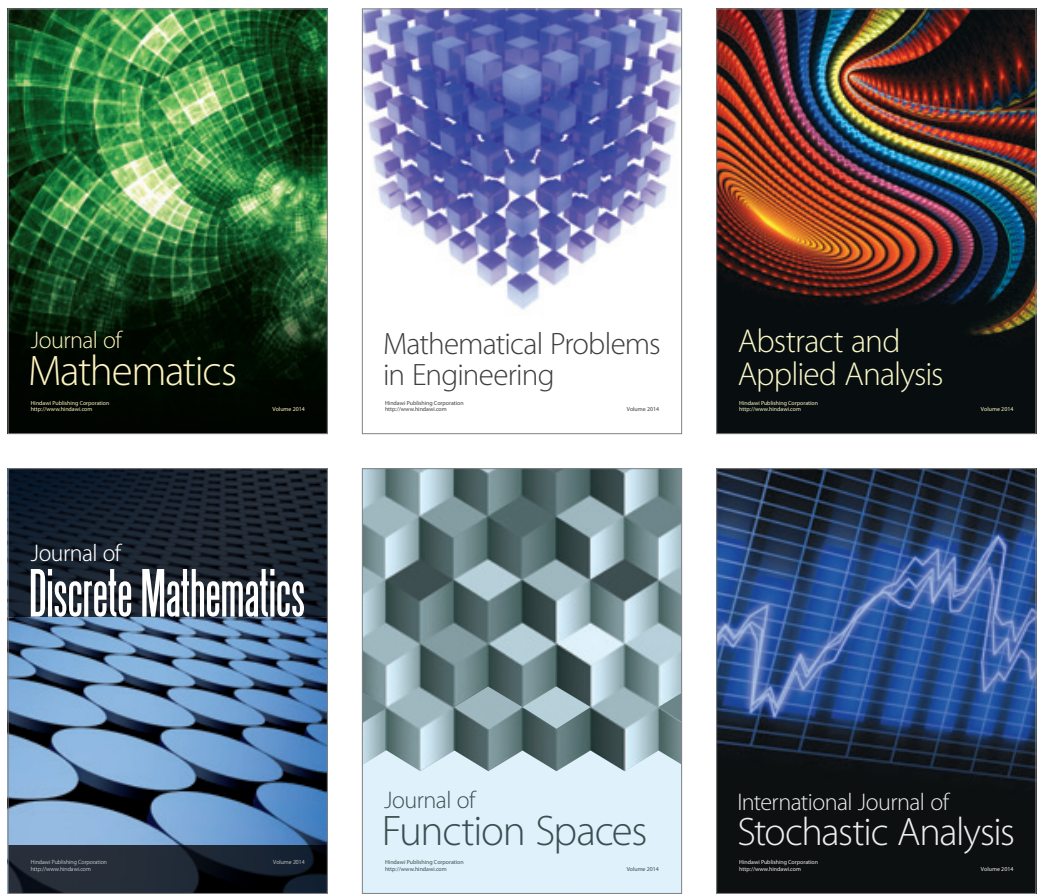

Journal of

Function Spaces

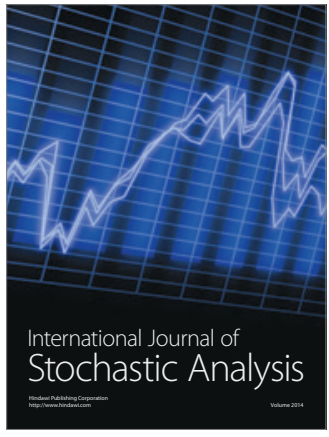

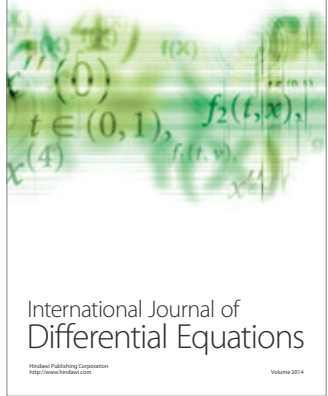
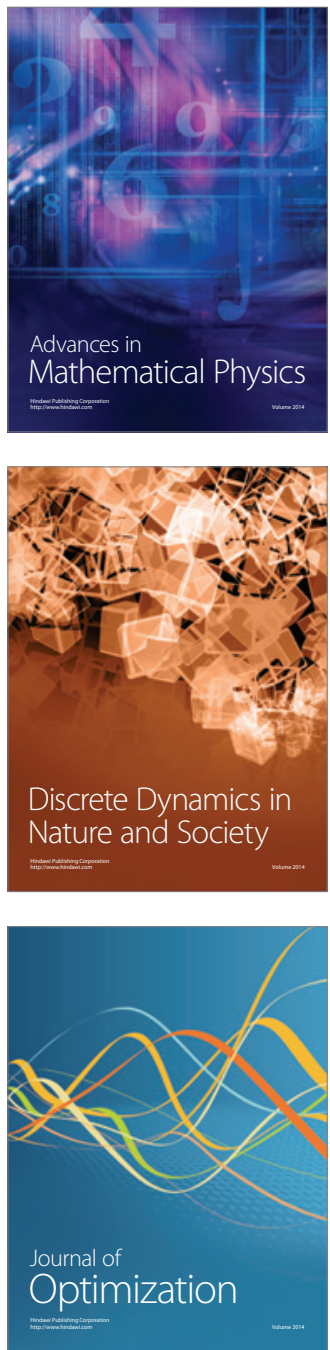